ARTICLE

\title{
Using ICRP/ICRU Voxel Models to Evaluate Specific Absorbed Fractions
}

\author{
Sakae KINASE $^{1 *}$, Masanori KIMURA ${ }^{1}$, Shogo TAKAHARA ${ }^{1}$ and Toshimitsu HOMMA ${ }^{1}$ \\ ${ }^{1}$ Nuclear Safety Research Center, Japan Atomic Energy Agency \\ 2-4 Shirane, Shirakata, Tokai-mura, Naka-gun, Ibaraki 319-1195, Japan
}

\begin{abstract}
The International Commission on Radiological Protection (ICRP) and the International Commission on Radiation Units and Measurements (ICRU) have developed reference computational models i.e. ICRP/ICRU voxel models of the Reference Male and Reference Female for calculations of reference dosimetric data, such as specific absorbed fractions (SAFs) - the fraction of energy emitted as a specified radiation type in a source organ that is absorbed per unit mass of target organ- for internal exposures. The task group on dose calculations (DOCAL) of ICRP committee 2 has evaluated SAFs for selected source and target organs in the ICRP/ICRU voxel models. However, published data on SAFs are limited. At the Japan Atomic Energy Agency, SAFs have been evaluated using voxel models and a Monte Carlo code, EGS4-UCSAF, to update a safety assessment code which provides doses after an accidental release of radioactive material to the atmosphere. In the present study, the ICRP/ICRU voxel models were applied to evaluating SAFs for both photons and electrons in organs of interest for internal dosimetry. The sources were assumed to be monoenergetic in the energy range from $10 \mathrm{keV}$ to $10 \mathrm{MeV}$. Consequently, it was confirmed that SAFs evaluated in the present study agree well with those evaluated by DOCAL, except for the cross-irradiation SAFs for electrons with low energies. The cross-irradiation SAF evaluations were found to be subject to the treatment of bremsstrahlung photons and the set of cutoff energies in the radiation transport.
\end{abstract}

\section{KEYWORDS: ICRP, voxel, SAF, dose, Monte Carlo simulation}

\section{Introduction}

Computational models such as voxel models of the human body have been the subject of many research papers ${ }^{1)}$, review articles $^{2)}$ and books ${ }^{3)}$. The ICRP/ICRU voxel models of the Reference Male and Reference Female given in ICRP Publication $89^{4)}$ have been developed by ICRP and ICRU. ${ }^{5)}$ The ICRP/ICRU voxel models are used for the evaluations of body-related protection quantities, SAFs for particles relevant to internal exposures. The first set of SAFs has been published by DOCAL of ICRP committee 2 using the ICRP/ICRU voxel models and Monte Carlo codes. ${ }^{5,6)}$ However, published data on SAFs have been confined to organs contributing to effective dose with the higher tissue weighting factors. At the Japan Atomic Energy Agency, SAFs have been evaluated using some human voxel models ${ }^{7)}$ and a Monte Carlo code to update a Level 3 probabilistic safety assessments (PSA) code which provides doses after an accidental release of radioactive material to the atmosphere. The present study was performed to evaluate SAFs for both photons and electrons in some organs using the ICRP/ICRU voxel models.

\section{Materials and Methods \\ 1. ICRP/ICRU voxel models}

The ICRP/ICRU adult male and female reference voxel models were used in the present study. The voxel models, based on computed tomographic data sets, were developed by

*Corresponding Author, E-mail:kinase.sakae@jaea.go.jp (C) 2012 Atomic Energy Society of Japan, All Rights Reserved.
DOCAL of ICRP committee 2 in collaboration with the Helmholtz Zentrum München and ICRU. The voxel sizes are $2.137 \times 2.137 \times 8.0 \mathrm{~mm}^{3}$ for the adult male voxel model and $1.775 \times 1.775 \times 4.84 \mathrm{~mm}^{3}$ for the adult female voxel model. The masses of the adrenals, kidneys, liver, lungs, pancreas and spleen for the voxel models are shown in Table 1. The defined organs are adjusted to approximate the reference mass values with high precision.

Table 1 Mass of each organ for the ICRP/ICRU voxel models.

\begin{tabular}{lcc}
\hline \multirow{2}{*}{ Organs } & \multicolumn{2}{c}{ Mass $(\mathrm{kg})$} \\
\cline { 2 - 3 } & Male & Female \\
\hline Adrenals & $1.4 \times 10^{-2}$ & $1.3 \times 10^{-2}$ \\
Kidneys & $3.1 \times 10^{-1}$ & $2.8 \times 10^{-1}$ \\
Liver & $1.8 \times 10^{0}$ & $1.4 \times 10^{0}$ \\
Lungs & $1.2 \times 10^{0}$ & $9.5 \times 10^{-1}$ \\
Pancreas & $1.4 \times 10^{-1}$ & $1.2 \times 10^{-1}$ \\
Spleen & $1.5 \times 10^{-1}$ & $1.3 \times 10^{-1}$ \\
\hline
\end{tabular}

\section{Specific absorbed fractions (SAFs)}

The specific absorbed fraction $\Phi$ is the absorbed fraction -the fraction of the energy emitted by source organ $r_{S}$ that is absorbed in target organ $\mathrm{r}_{\mathrm{T}^{-}}$in the target per unit mass of the target. It can be expressed by the equation:

$$
\Phi_{\mathrm{i}}\left(\mathrm{r}_{\mathrm{T}} \leftarrow \mathrm{r}_{\mathrm{S}}\right)=\frac{1}{\mathrm{~m}_{\mathrm{T}}} \cdot \frac{\begin{array}{c}
\text { i-type radiation energy } \\
\text { absorbed in target } \mathrm{r}_{\mathrm{T}}
\end{array}}{\begin{array}{c}
\text { i-type radiation energy } \\
\text { emitted in source } \mathrm{r}_{\mathrm{S}}
\end{array}}
$$


Specific absorbed fractions for photons and electrons were evaluated for the ICRP/ICRU adult male and female reference voxel models using the Monte Carlo code, EGS4 ${ }^{8)}$, in conjunction with an EGS4 user code, $\mathrm{UCSAF}^{7}$. In the EGS4-UCSAF code, the radiation transport of electrons, positrons and photons in the phantoms was simulated, and correlations between primary and secondary particles are included. In the present study, the sources of the photons and electrons were assumed to be monoenergetic in the energy range from $10 \mathrm{keV}$ to $10 \mathrm{MeV}$ and uniformly distributed in the source organ. The source organs were the adrenals, kidneys, liver, lungs, pancreas and spleen. Photon and electron histories were selected to be numbers sufficient to reduce statistical uncertainties below 5\%. The cutoff energies were set to $1 \mathrm{keV}$ for the photons and $10 \mathrm{keV}$ for the electrons. The Parameter Reduced Electron-Step Transport Algorithm (PRESTA) ${ }^{9)}$ to improve the electron transport in the low-energy region was used. The cross-section data for photons were taken from PHOTX $^{10)}$ and the data for electrons are taken from ICRU report $37^{11)}$.

\section{Results and Discussion}

\section{Self-irradiation SAFs}

Figure 1 shows self-irradiation SAFs for photons and electrons in the lungs of the ICRP/ICRU adult male reference voxel model in the energy range from $10 \mathrm{keV}$ to $10 \mathrm{MeV}$.

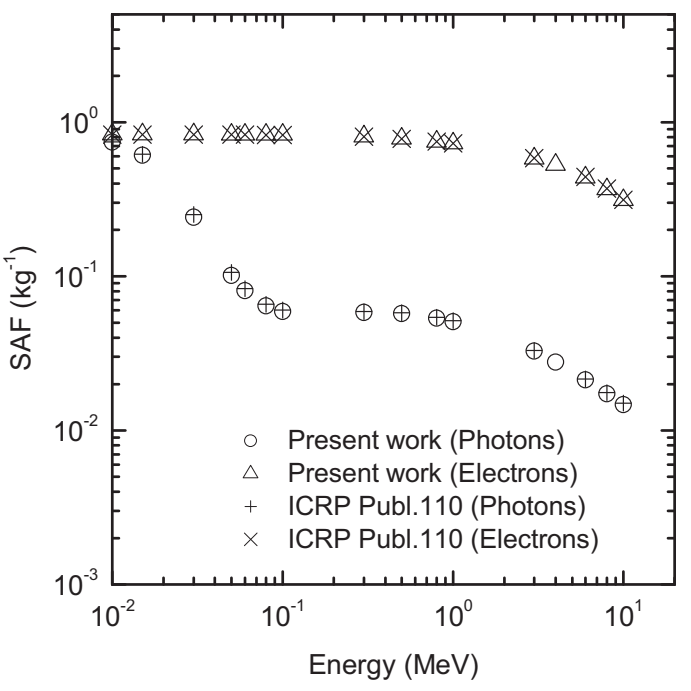

Fig. 1 Self-irradiation specific absorbed fractions in the lungs of the ICRP/ICRU adult male voxel model.

The self-irradiation SAF for photons decreases with an increase in photon energy on the whole. The self-irradiation SAF for electrons shows constancy up to several hundreds $\mathrm{keV}$ and then a decrease. The self-irradiation SAFs for photons and electrons in the lungs of the ICRP/ICRU adult male reference voxel model depend on the radiation energy.

Table 2 Self-irradiation specific absorbed fractions in some organs for ICRP/ICRU voxel models, Otoko ${ }^{7)}$, Onago ${ }^{7)}$, MIRD 5 type ${ }^{12)}$ model for photon/electron energies of $30 \mathrm{keV}, 100 \mathrm{keV}$ and $1 \mathrm{MeV}$.

\begin{tabular}{|c|c|c|c|c|c|c|c|}
\hline \multirow{2}{*}{ Organ } & \multicolumn{5}{|c|}{$\begin{array}{l}\text { Specific absorbed fraction }\left(\mathrm{kg}^{-1}\right) \\
\text { for photons }\end{array}$} & \multicolumn{2}{|c|}{$\begin{array}{l}\text { Specific absorbed fraction }\left(\mathrm{kg}^{-1}\right) \\
\text { for electrons }\end{array}$} \\
\hline & $\begin{array}{l}\text { ICRP/ICRU } \\
\text { male }\end{array}$ & $\begin{array}{l}\text { ICRP/ICRU } \\
\text { female }\end{array}$ & Otoko $^{7)}$ & Onago ${ }^{7)}$ & MIRD $^{12)}$ & $\begin{array}{l}\text { ICRP/ICRU } \\
\text { male }\end{array}$ & $\begin{array}{l}\text { ICRP/ICRU } \\
\text { female }\end{array}$ \\
\hline \multicolumn{8}{|c|}{ Energy, $30 \mathrm{keV}$} \\
\hline Adrenals & 6.7 & 9.1 & 5.7 & 6.3 & 6.8 & 71 & 77 \\
\hline Kidneys & 1.2 & 1.3 & 1.2 & 1.2 & 0.99 & 3.2 & 3.6 \\
\hline Liver & 0.33 & 0.39 & 0.43 & 0.38 & 0.28 & 0.56 & 0.71 \\
\hline Lungs & 0.29 & 0.22 & 0.18 & 0.24 & 0.24 & 0.83 & 1.1 \\
\hline Pancreas & 2.3 & 2.3 & 2.4 & 4.1 & 2.4 & 7.1 & 8.3 \\
\hline Spleen & 2.3 & 2.4 & 3.4 & 3.0 & 1.8 & 6.7 & 7.7 \\
\hline \multicolumn{8}{|c|}{ Energy, $100 \mathrm{keV}$} \\
\hline Adrenals & 1.2 & 1.6 & 1.0 & 1.1 & 1.3 & 70 & 76 \\
\hline Kidneys & 0.24 & 0.27 & 0.26 & 0.26 & 0.23 & 3.2 & 3.6 \\
\hline Liver & 0.10 & 0.11 & 0.12 & 0.11 & 0.092 & 0.55 & 0.71 \\
\hline Lungs & 0.059 & 0.070 & 0.041 & 0.055 & 0.053 & 0.83 & 1.0 \\
\hline Pancreas & 0.48 & 0.47 & 0.51 & 0.81 & 0.52 & 7.1 & 8.3 \\
\hline Spleen & 0.47 & 0.48 & 0.68 & 0.61 & 0.42 & 6.6 & 7.7 \\
\hline \multicolumn{8}{|c|}{ Energy, $1 \mathrm{MeV}$} \\
\hline Adrenals & 1.2 & 1.6 & 1.0 & 1.1 & 1.5 & 49 & 61 \\
\hline Kidneys & 0.22 & 0.25 & 0.25 & 0.25 & 0.23 & 3.0 & 3.4 \\
\hline Liver & 0.084 & 0.097 & 0.11 & 0.098 & 0.081 & 0.54 & 0.69 \\
\hline Lungs & 0.051 & 0.061 & 0.036 & 0.048 & 0.047 & 0.73 & 0.94 \\
\hline Pancreas & 0.45 & 0.45 & 0.48 & 0.79 & 0.53 & 6.6 & 7.5 \\
\hline Spleen & 0.44 & 0.45 & 0.68 & 0.59 & 0.41 & 6.2 & 7.0 \\
\hline
\end{tabular}


In Figure 1, the self-irradiation SAFs published by DOCAL of ICRP committee 2 are also plotted for comparison. It can be seen from the figure that the self-irradiation SAFs evaluated in the present study are in good agreement with those evaluated by ICRP. Hence, the results support that the EGS4-UCSAF code can be used to evaluate self-irradiation SAFs for photons and electrons in the ICRP/ICRU voxel models.

Self-irradiation SAFs for photons and electrons in some organs of the ICRP/ICRU adult male and female reference voxel models are illustrated in Table 2 , where previous works using Japanese adult male and female voxel models (Otoko and Onago), MIRD 5 type model are listed. As mentioned in the previous studies, the self-irradiation SAF depends on the mass of the target. The result shows that the self-irradiation SAF for photons is not significantly changed among different human models.

\section{Cross-irradiation SAFs}

Cross-irradiation SAFs for photons and electrons in the ICRP/ICRU adult female reference voxel model are plotted against radiation energy in Figure 2. The source organ is liver and the target organ is stomach wall (the mass of the stomach wall is $1.4 \times 10^{-1} \mathrm{~kg}$ for the voxel model). The cross-irradiation SAF for photons increases up to $30 \mathrm{keV}$ and then is followed by a gradual decrease. The cross-irradiation SAF for electrons shows an abrupt increase up to several $\mathrm{MeV}$. Figure 2 provides that the cross-irradiation SAFs evaluated in the present study agree exactly with those evaluated by DOCAL of ICRP committee 2. The part confirms that the EGS4-UCSAF code is useful for evaluating the cross-irradiation SAFs for photons and electrons in the ICRP/ICRU voxel models. However, it must be emphasized that the cross-irradiation SAFs for electrons with low energies are different from those evaluated by

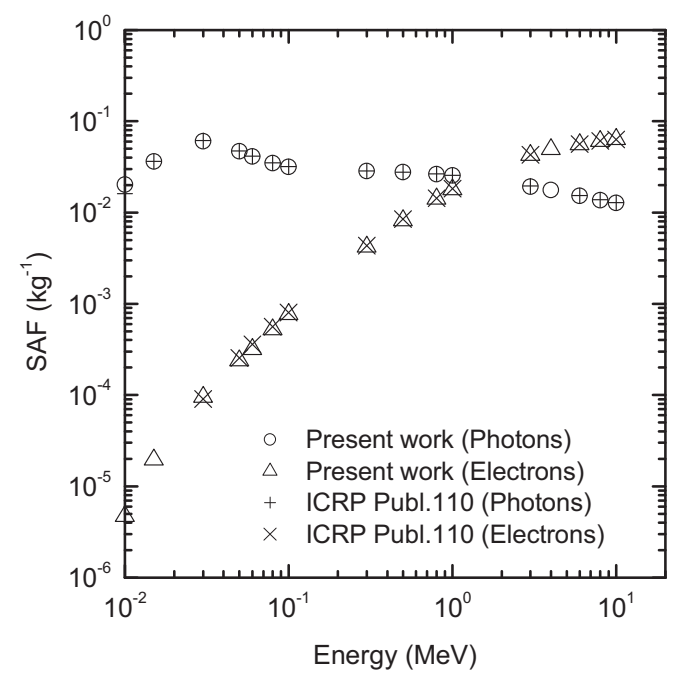

Fig. 2 Cross-irradiation specific absorbed fractions for source organ liver and target organ stomach wall in the ICRP/ICRU adult female voxel model.
ICRP. The cross-irradiation SAFs for electrons with low energies at 10, $15 \mathrm{keV}$ have been replaced with zero in DOCAL of ICRP committee 2. In the Monte Carlo simulations by ICRP, the cutoff energies were $2 \mathrm{keV}$ for photons and $20 \mathrm{keV}$ for electrons; for primary electron energies below $50 \mathrm{keV}$, an electron cutoff of $2 \mathrm{keV}$ was used. These discrepancies are thought to be due to the set of cutoff energies and the treatment of bremsstrahlung photons in the radiation transport.

\section{Conclusions}

Specific absorbed fractions for photons and electrons were evaluated for the ICRP/ICRU voxel models using the EGS4-UCSAF code. Good agreement between the SAFs evaluated in the present study and those evaluated by ICRP is shown. The present study showed that the self-irradiation SAF depends on the mass of the target and that the set of cutoff energies and the treatment of bremsstrahlung photons should be considered with discretion in the radiation transport.

\section{References}

1) M. Zankl, R. Veit, G. Williams, et al., The construction of computer tomographic phantoms and their application in radiology and radiation protection, Radiat. Environ. Biophys. 27 (1988), 153-164.

2) H. Zaidi and B. M. W. Tsui, Review of computational anthropomorphic anatomical and physiological models, Proc. IEEE 97 (2009), 1938-1953.

3) X. G. Xu and K. F. Eckerman, Handbook of anatomical models for radiation dosimetry, CRC Press, New York (2009), ISBN 978-1-4200-5979-3.

4) ICRP, Basic anatomical and physiological data for use in radiological protection: reference values, ICRP Publication 89 International Commission on Radiological Protection (2002).

5) ICRP, Adult reference computational phantoms, ICRP Publication 110 International Commission on Radiological Protection (2009).

6) L. Hadid, A. Desbrée, H. Schlatti, et al., Application of the ICRP/ICRU reference computational phantoms to internal dosimetry: calculation of specific absorbed fractions of energy for photons and electrons, Phys. Med. Biol. 55 (2010), 3631-3641.

7) S. Kinase, M. Zankl, J. Kuwabara, et al., Evaluation of specific absorbed fractions in voxel phantoms using Monte Carlo simulation, Radiat. Prot. Dosim. 105 (2003), 557-563.

8) W. R. Nelson, H. Hirayama and D. W. O. Rogers, The EGS4 Code System, SLAC-265 (1985).

9) A. F. Bielajew and D. W. O. Rogers, Electron step-size artefacts and PRESTA, in Monte Carlo Transport of Electrons and Photons, T. M. Jenkins, W. R. Nelson, and A. Rindi, Eds. Plenum Press, New York (1987), ISBN 0-306-43099-1.

10) NIST, Photon Interaction Cross Section Library, DLC-136/PHOTX, contributed by National Institute of Standards and Technology (1989).

11) ICRU, Stopping Powers for Electrons and Positrons, ICRU Report 37 International Commission on Radiation Units and Measurements (1984).

12) M. Cristy and K. F. Eckerman, Specific absorbed fractions of energy at various ages from internal photon sources, ORNL/TM-8381 Vol. 1-7 (Oak Ridge National Laboratory, Oak Ridge, Tennessee) (1987). 